\title{
Effect of Thermo Chemical Processing on Storability of Sugarcane Juice
}

\author{
Bonny Mathew, Mini.V., Jessy M. Kuriakose, Shajan V.R., Jayakumar G.
}

Agricultural Research Station, Kerala Agricultural University, Thiruvalla, Kerala, India

\begin{abstract}
A study has been undertaken at Agricultural Research Station, Thiruvalla with an objective to study the effect of thermo chemical processing on storability of sugarcane juice to develop a protocol for preservation of sugarcane juice. Fresh sugarcane juice was subjected to ten different treatments. Juice under different treatments was analyzed before preservation and after preservation. The scores for sensory qualities (colour, taste, smell and overall acceptability) reduced significantly with the advancement of storage. Change in physico- chemical characteristics ( $\mathrm{pH}$, TSS, titratable acidity and Brix: acid ratio) also followed the same decreasing trend during storage period. The treatment $T 5$ recorded the highest scores in sensory evaluation and it recorded the highest Brix: acid ratio after the storage period, indicating better consumer acceptability. The results revealed that good quality beverage from sugarcane juice with satisfactory storage stability of 30 days at refrigeration could be prepared by adding $0.5 \mathrm{ml}$ lime juice, $0.2 \mathrm{ml}$ ginger extract and $125 \mathrm{ppm}$ of sodium meta bisulphite per $100 \mathrm{ml}$ of sugarcane juice and microwave pasteurization of the treated juice for one minute. This protocol for preservation of sugarcane juice with microwave pasteurization and preservatives will be very useful for commercial preservation of sugarcane juice.
\end{abstract} Keywords-Sugarcane juice, Microwave pasteurization, shelf life, sensory evaluation.

\section{INTRODUCTION}

Sugarcane juice is a very popular drink in India but still it is rarely available commercially in packaged form. Development of effective treatments or procedures to keep the fresh quality of sugarcane juice would allow it to be more widely marketed, and would enhance its quality and safety as well. Sugarcane juice has many medicinal properties, often it is used as a remedy for jaundice in traditional medicine (Subbannayya et al., 2007). Even the diabetic can enjoy this sweet drink without fear because it has no simple sugars. It hydrates the body quickly when exposed to prolong heat and physical activity. It is an excellent substitute for aerated drinks and cola and it refreshes and energizes the body instantly as it is rich in carbohydrates. If the cane juice is collected and preserved in a hygienic way then it can be sold after short or long time preservation. It will meet up the nutritional problem of our large population.

Darkening of sugarcane juice occurs by formation of brown pigments by enzymatic and non-enzymatic reactions. Boiling of sugarcane juice for preservation will cause browning of sugarcane juice and jaggery flavour will be developed. Therefore thermal processing at higher temperature is not possible in the case of sugarcane juice. In circumstances where thermal processing is impractical, minimal processing employing different barriers to microbial growth can enhance product stability. Thus good sanitation is the first barrier to reduce microbial load and low storage temperature further retards growth. An acid environment of $\mathrm{pH}$ less than 4.5 restricts the growth of many organisms. Mostly fruit juices are acidic and can be used for acidification of sugarcane juice. The exclusion of oxygen is an additional barrier. Antimicrobial substances, either natural or chemical preservatives, also assist. It has been observed that low temperature storage is able to extend the shelf life of the juice for a few days. However, no study has been conducted to elaborate these parameters.

Many scientists' viz., Prati et al. (2005) and Rezzadori (2010) reported that sugarcane juice pasteurization can be done with and without the addition of fruit juices. But the impact of microwave pasteurization and preservatives on the sensory stability of sugarcane juice has not yet been addressed. Keeping all these points in view, the present study was undertaken to study the effect of thermo chemical processing on storability of sugarcane juice to develop a protocol for preservation of sugarcane juice

\section{MATERIALS AND METHODS}

The present investigation was carried out at Agricultural Research Station, Thiruvalla and the sugarcane variety Madhuri was used for the extraction of sugarcane juice. Fresh sugarcane stems were used for the juice recovery process. Graded sample were washed by running tap water 
to get sugarcane free from any dust and dirt. The skin and node of sugarcane stem were removed with the help of curved blade knife. Sugarcane juice was extracted by power operated juice extractor and filtered through the sieve and muslin cloth to remove the extraneous matter. Fresh sugarcane juice after initial microwave treatment at $70^{\circ} \mathrm{C}$ for 30 seconds was taken for further treatments viz., T1 (Microwave pasteurization for 30 seconds),T2 (Juice + Citric acid @ 30mg/100ml followed by Microwave pasteurization for 30 seconds), T3 (Juice+ Citric acid @ 30mg/100ml+ KMS @ 125ppm followed by Microwave pasteurization for 30 seconds), T4 (Juice+0.3\% lemon juice $+0.1 \%$ ginger $+100 \mathrm{ppm}$ KMS followed by microwave pasteurization for 30 seconds),T5(Juice+0.5\% lemon juice $+0.2 \%$ ginger $+125 \mathrm{ppm}$ Sodium Meta bi sulphite followed by Microwave pasteurization for 1 minute), T6 ( Boiling the juice and hot bottling), T7 (Juice+ Ascorbic acid @ 30mg/100ml followed by Microwave pasteurization for 30 seconds),T8 (Juice+ Ascorbic acid @ 30mg/100ml+
KMS @ $125 \mathrm{ppm}$ followed by microwave pasteurization for 30 seconds), T9 (Juice $+1 \%$ lemon juice $+0.3 \%$ ginger $+0.25 \mathrm{~g}$ salt+ $75 \mathrm{ppm}$ KMS followed by Microwave pasteurization for 30 seconds), T10 (Control (keep in refrigerated condition). Juice under different treatments were analysed before preservation and after preservation. The sugarcane juice samples were analysed for total soluble solids, $\mathrm{pH}$ and titratable acidity at 15 days interval by the methods described by Ranganna (1995). From the brix value and acidity percentage, Brix/ Acid ratio was also worked out to predict consumer acceptability. Sensory evaluation was also done for juice under different treatments.

\section{RESULTS AND DISCUSSION}

Physico-Chemical Characteristics

Physico chemical properties of sugarcane juice under different treatments were studied at 15 days interval up to one month. The results are presented in Table 1.

Table.1: Physico-Chemical characteristics of sugarcane juice under different treatments

\begin{tabular}{|c|c|c|c|c|c|c|c|c|c|c|c|c|}
\hline \multirow[t]{3}{*}{ Treatments } & \multicolumn{3}{|c|}{$\mathrm{pH}$} & \multicolumn{3}{|c|}{ TSS (॰ brix) } & \multicolumn{3}{|c|}{ Acidity (\%) } & \multicolumn{3}{|c|}{ Brix/ Acid ratio } \\
\hline & Day & Day & Day & Day & Day & Day & Day & Day & Day & Day & Day 15 & Day \\
\hline & 1 & 15 & 30 & 1 & 15 & 30 & 1 & 15 & 30 & 1 & & 30 \\
\hline $\mathrm{T} 1$ & 4.4 & 4.26 & 3.8 & 19 & 18 & 17 & 0.04 & 0.05 & 0.06 & 475 & 360 & 283 \\
\hline $\mathrm{T} 2$ & 4.14 & 3.8 & 3.39 & 19 & 19 & 16 & 0.04 & 0.05 & 0.06 & 475 & 380 & 267 \\
\hline T3 & 4.27 & 4.09 & 3.97 & 17 & 17 & 16 & 0.04 & 0.05 & 0.05 & 425 & 340 & 320 \\
\hline $\mathrm{T} 4$ & 4.09 & 4.03 & 3.95 & 18 & 17 & 17 & 0.04 & 0.05 & 0.06 & 450 & 340 & 283 \\
\hline T5 & 4.27 & 4.18 & 4.11 & 20 & 19 & 19 & 0.04 & 0.05 & 0.05 & 500 & 380 & 380 \\
\hline T6 & 4.07 & 3.84 & 3.64 & 19 & 18 & 17 & 0.04 & 0.06 & 0.07 & 475 & 300 & 243 \\
\hline $\mathrm{T} 7$ & 4.01 & 3.65 & 3.46 & 18 & 17 & 15 & 0.04 & 0.07 & 0.08 & 450 & 243 & 187 \\
\hline $\mathrm{T} 8$ & 4.27 & 4.01 & 3.23 & 19 & 18 & 16 & 0.04 & 0.05 & 0.09 & 450 & 360 & 178 \\
\hline T9 & 4.28 & 3.88 & 3.39 & 19 & 18 & 15 & 0.04 & 0.06 & 0.07 & 450 & 300 & 214 \\
\hline T10 & 4.03 & 3.65 & 3.22 & 20 & 18 & 14 & 0.04 & 0.07 & 0.08 & 500 & 257 & 175 \\
\hline
\end{tabular}

\section{Total soluble solids}

The total soluble solids decreased during storage of sugarcane juice. These observations are in agreement with the findings of Bhupinder et al. (1991). They attributed the decrease in total soluble solids to breakdown of sugars to acids during storage as a result of action of microorganism present in the juice. The decrease was lowest in treatment T5 (Table 1). Addition of lime juice and microwave pasteurization of sugarcane juice restricted the degradation of total soluble solids and total sugars during storage. Citric acid in lime juice acted as a natural preservative and helped in the restriction of degradation of total soluble solids. Addition of sodium meta bisulphite to the juices further reduced the extent of changes in total soluble solids and sugars due to the suppression of microbial activity

\section{pH and titratable acidity}

The $\mathrm{pH}$ decreased whereas acidity increased during storage of sugarcane juice (Table 1). Addition of organic acids to juice also increased its acidity and decreased $\mathrm{pH}$. Addition of sodium meta bisulphite to juice restricted the microbial activity during storage resulting in less reduction in $\mathrm{pH}$ and less increase in acidity of treatment $\mathrm{T} 5$. The reduction in $\mathrm{pH}$ and increase in acidity was, however, higher in the control. Acidity of juice increased with storage time. The increase in acidity caused a concomitant decrease in $\mathrm{pH}$ value. A similar result reported by Abbo et al. (2006) 
revealed that there is a corresponding reduction in $\mathrm{pH}$ as the acidity increased in sour soup juice. The reason for high acid and low pH could be due to acetic acid and lactic acid production.

\section{Brix: Acid ratio}

Based on the Brix reading and titratable acidity percentage, Brix: acid ratio was worked out to find the overall acceptability. There was a considerable reduction in the Brix: acid ratio during the storage period.

In treatment $\mathrm{T} 5$ and control, the initial Brix: acid ratio was 500 and in the control it drastically reduced to 175 at the end of 30 days after storage (Table 1). This is due to the change in total soluble solids and acidity during storage. The highest Brix: acid ratio at the end of storage period was recorded by treatment $\mathrm{T} 5$, indicating highest overall acceptability compared to all other treatments. Bhupinder et al. (1991) reported that addition of lemon and ginger followed by pasteurization and preservation with sulphur dioxide reduced physico-chemical changes during storage of sugarcane juice.

\section{Sensory evaluation}

The sugarcane juice under different treatments was awarded sensory scores ranging between 1 to 9 for colour, taste, smell, sweetness and overall acceptability by the panelists.

\section{Colour}

The sensory scores for colour reduced significantly with the advancement of storage (Fig.1). However the reduction in sensory scores of treatments T5 was of lesser magnitude than other treatments. The lowest reduction in sensory scores was observed in sugarcane juice pasteurized after the addition of $0.5 \%$ lemon juice, $0.2 \%$ ginger extract and 125 ppm Sodium metabisulphite. In this treatment microwave pasteurization was done for one minute. In control sample the sensory colour score after 15 days was found 3 because of browning observed in the control with a rapid decrease in colour. Browning occurred in sugarcane juice due to increasing PPO (Polyphenol oxidase) activity and invert sugar. The colour of sugarcane juice became darker and it decreased its appearance score (Plate 1). Similar result was found by Mao et al. (2007) during the study of preservation of sugarcane juice.

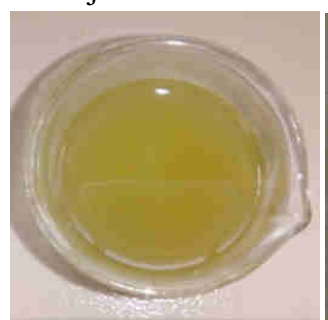

T5 (Best treatment)

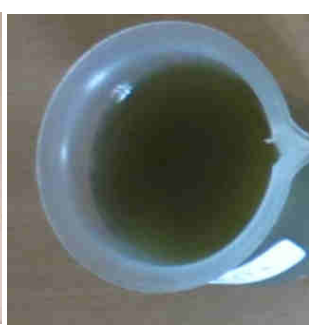

T10 (control)
Plate.1: Appearnce of T5 and T10 after 30 days of storage period

\section{Taste}

Reduction in the sensory scores for taste with the advancement of storage is evident from the (Fig.2). Treatment $\mathrm{T} 5$ recorded the lowest reduction in sensory scores for taste. In all other treatments the taste was changed considerably during the storage period. In control sample the taste score was decreased from 6 to 2 after 30 days and this decrease could be due to the loss of volatile aromatic substances responsible for taste and due to decreases in $\mathrm{pH}$ of the juice as it became more acidic as reported by Reddy (2004).

\section{Smell}

The sensory scores for smell reduced with the advancement of storage period (Fig.3). The score values for smell decreased during storage of sugarcane juice between 0 to 30 days. In control sample the score was decreased from 6 to 3 after 30 days. This decrease could be due to high level of acid that reacts with the product and results in unpleasant volatile odour and this could be due to the slight fermentation of juice and gas production. There has been significant decline in score of smell of sugarcane juice. Reddy (2004) also found similar results and he reported that the loss of volatile aromatic substances is responsible for the decrease in score of smell. Treatment T4 and T5 recorded lower reduction in scores and T5 ( Micro wave pasteurization for one minute after addition of $0.5 \%$ lemon juice, $0.2 \%$ ginger extract and $125 \mathrm{ppm}$ Sodium meta bi sulphite) recorded the highest score even after 30 days of storage period. Micro wave pasteurization and addition of lime juice and sodium meta bisulphite had prevented changes in smell in the treatment T5.

\section{Sweetness}

There was a considerable reduction in the sensory scores for sweetness with the advancement of storage period (Fig.4). Initially treatments $\mathrm{T} 4, \mathrm{~T} 5$ and $\mathrm{T} 10$ recorded the highest score (9) for sweetness. Later the sweetness got reduced and the reduction was very rapid under control and at the end of 15 days after treatment, T5 recorded the highest score and it retained the score even up to 30 days.

\section{Overall acceptability}

Reduction in the sensory scores for overall acceptability is evident from the Fig.5. Treatment T5 recorded the highest sensory scores for overall acceptability at the end of storage period. There were not much changes in colour, taste, smell and appearance in treatment T5. In control sample the overall acceptability was 8 on the first day and it decreased to 3 at the end of storage period. Score of control sample declined during storage due to the oxidative reaction which deteriorated the scores of colour, smell, appearance as well as taste. These findings were in accordance with Chauhan $e t$ 
al. (2002) .This decrease could be due to the fermentation of juice and gas production.

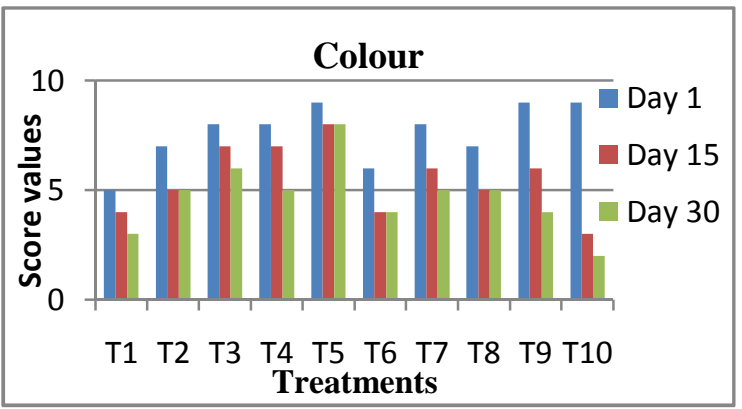

Fig.1: Change in colour of sugarcane juice

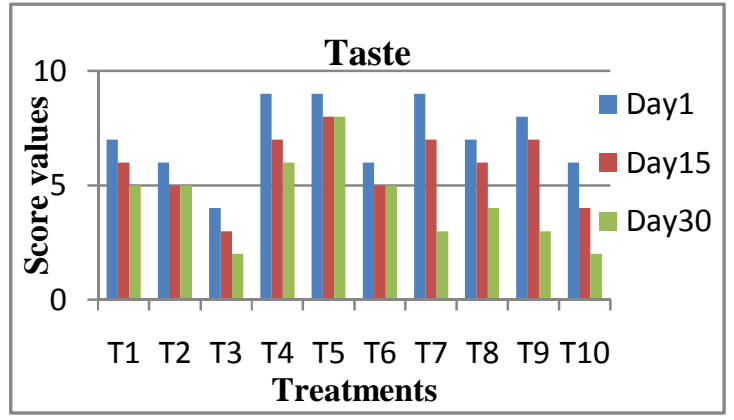

Fig.2: Change in taste of sugarcane juice

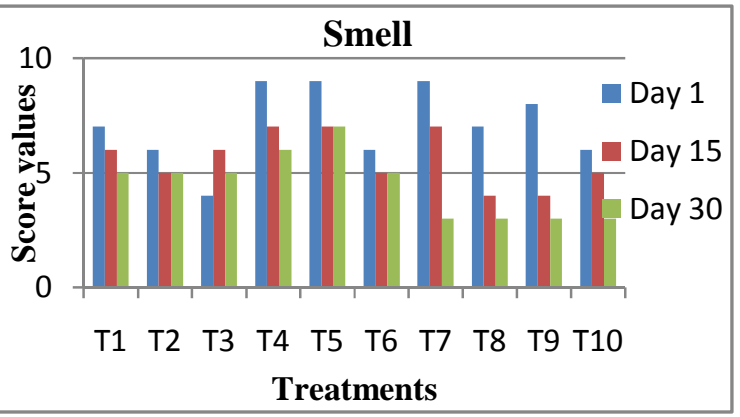

Fig.3: Change in smell of sugarcane juice

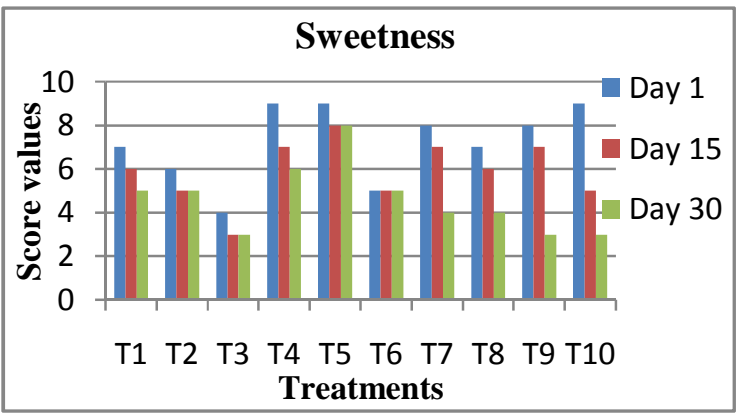

Fig.4: Change in sweetness of sugarcane juice

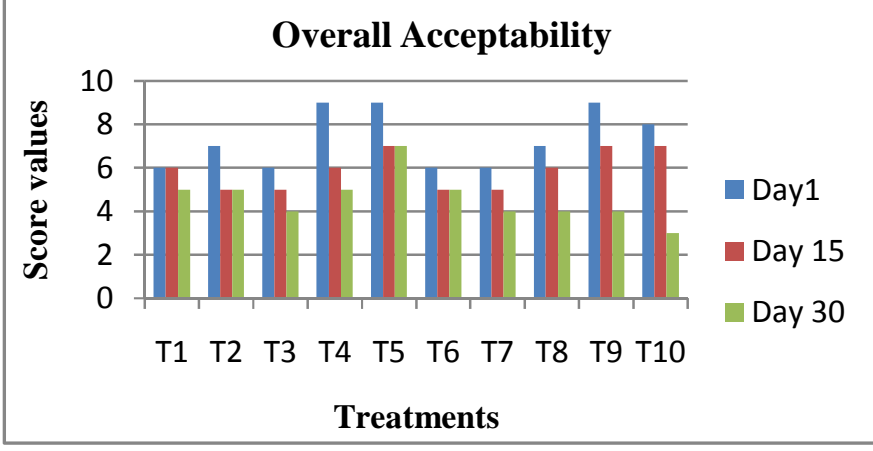

Fig.5: Change in overall acceptability of sugarcane juice

\section{Conclusion}

The treatment T5 (micro wave pasteurization for one minute after addition of $0.5 \%$ lemon juice, $0.2 \%$ ginger extract and $125 \mathrm{ppm}$ Sodium meta bisulphite) recorded the highest scores in sensory evaluation also. The citric acid in lime juice gave a preservative action and inhibited the growth of micro-organism during storage. Sodium metabisulphite is also a known yeast and mold inhibitor and is being used widely for the preservation of foods. This protocol for preservation of sugarcane juice with microwave pasteurization and preservatives will be very useful for preservation of sugarcane juice and the sugarcane juice can be introduced as delicious beverages by preventing the spoilage of juice with this technology.

\section{REFERENCES}

[1] Abbo, E.S., Odeyemi, G. and Glurius. T.O. 2006. Studies on the storage stability of sour soup juice. African Journal of Microbiology 21: 197-214.

[2] Bhupinder,K., Sharma,K.P. and Harinder,K.1991. Studies on the development and storage stability for ready to serve bottled sugarcane juice, Int. J. Tropical Agric, 9:128-134.

[3] Chauhan, O. P., Singh,D., Tyagi,S.M. and Balyan, D.K. 2002. Studies on preservation of sugarcane juice. Int. J. Food Properties 5 : 217-229.

[4] Mao,L.C. QuanXu,Y. and Que,F. 2007. Maintaining the quality of sugarcane juice with blanching and ascorbic acid. Food Chem. 104: 740-745.

[5] Prati, P., Moretti, R. H. and Cardello, M. A. B. 2005."Elaboration ofbeverage composed by blends of clarified-stabilized sugarcane juice and juice's acid fruits", Food Sci.Technol. 25: 147-152.

[6] Ranganna, S. 1995. Handbook of analysis and quality control for fruits and vegetable products. Tata McGraw-Hill Publishing Company Limited, New Delhi, India. 
[7] Reddy, G.N.V.V. 2004. Studies on methods on extraction of sapota juice for optimum yield and quality. MSc. Thesis submitted to Acharya N. G. Ranga Agricultural University, Hyderabad.

[8] Rezzadori, K. 2010. Thermal pasteurization with membranes of sugarcane juice with added passion fruit juice. Florianópolis: Federal University of Santa Catarina.

[9] Subbannayya, K., Bhat G.K., Shetty, S. and Junu, V.G. 2007. How safe is sugarcane juice. Indian $J$. Med. Microbiol.25:73-74. 